\title{
Investigation of the physical mechanisms of shear-force imaging
}

\author{
C. Durkan and I. V. Shvets \\ Department of Physics, Trinity College, Dublin 2, Ireland
}

(Received 4 June 1996; accepted for publication 13 August 1996)

\begin{abstract}
It is shown that shear-force imaging, as is commonly used for distance regulation in scanning near-field optical microscopy, is not a reliable technique for accurate topographic measurements. This is because different materials experience different shear-force damping. Results of the shear-force damping characteristics are presented for a number of different materials, and some consequences of the different dampings for different materials are demonstrated. It is also shown that there are at least two distinct shear force damping mechanisms. Results of imaging small conducting islands on a glass substrate show that the damping characteristics depend on the islands' size. (C) 1996 American Institute of Physics. [S0021-8979(96)02722-3]
\end{abstract}

\section{INTRODUCTION}

Scanning near-field optical microscopy (SNOM) has become an intensively developed area in optics. ${ }^{1-3}$ The technique allows optical imaging with resolution down to 40-60 $\mathrm{nm}$ or better, well below the diffraction limit. ${ }^{4}$ The principle of SNOM is that a probe, usually consisting of a tapered single-mode optical fiber is metal coated in such a way as to leave a small aperture of diameter $50-150 \mathrm{~nm}$ at the tip apex. The fiber scans over a given sample surface and the light coupled into the fiber forms a submicron light source at the aperture. The distance between the end of the fiber and the surface should be less than the aperture radius to achieve such a high optical resolution. ${ }^{5}$ The preferred distance control mechanism maintaining a constant separation between the end of the tip and the sample is based on shear-force detection. ${ }^{6}$

The principle of shear-force is that if a fiber tip is oscillated near mechanical resonance $(10-200 \mathrm{kHz})$ by a few $\mathrm{nm}$, then on approach to a sample surface, within a distance of a few $\mathrm{nm}$, these oscillations become damped out. The underlying mechanism is not fully understood, but a number of possibilities have been put forward, including long-range van der Waals forces, image charge current dissipation, viscous damping in a contaminant layer, and short-range mechanical contact (knocking). ${ }^{7}$

Shear-force distance control is of fundamental importance for SNOM imaging. Malfunctioning of the mechanism inevitably leads to artifacts in optical images. Due to the high confinement of light in the vicinity of an SNOM aperture, variations as small as a few $\AA$ in tip-sample separation can result in changes in the optical signal of up to a few percent. A number of factors which can typically cause malfunctioning of the shear-force distance control mechanism were studied recently by the authors. ${ }^{8}$ They include broadening of the lateral size of the surface features due to the dithering of the tip, effects caused by trapping an SNOM fiber in depressions on a surface, and dynamic effects due to the feedback mechanism. In this work, we concentrate on more fundamental aspects of the shear-force distance control. We investigate some of the possible physical origins of the shear-force and demonstrate that even an "ideally" working shear-force distance regulation system will still inevitably malfunction in certain experimental situations, most notably when imaging samples composed of different materials.

\section{SHEAR-FORCE MICROSCOPE}

The shear-force microscope is engineered as shown in Fig. 1. For imaging involving uncoated fiber tips, the detection beam from the optical fiber $D$ (detection fiber) is passed directly through the SNOM fiber shaft $t$, just before the tapered region of the tip, and is collected by a lens $l$ attached to a photodiode. This is essentially a beam deflection method. With this arrangement, we can work with SNOM fiber oscillation amplitudes of 2-3 nm peak-peak. For imaging involving tips coated with aluminum, the more conventional edge-shadowing technique is employed. ${ }^{9}$ It allows us to work with fiber oscillation amplitudes of 5-10 nm peak-peak. The same setup may be used for both experimental situations.

In order to characterize the performance of the shearforce microscope, a test sample was imaged. This was a carbon cross-grating consisting of a series of v-shaped grooves, of period $460 \mathrm{~nm}$, groove width $80-100 \mathrm{~nm}$, and groove depth $40-80 \mathrm{~nm} .{ }^{10}$ Figure 2 shows a shear-force image of this sample taken with a coated optical fiber tip using the edge-shadowing shear-force detection scheme, showing that high resolution images may be obtained even with large tips. The metal coating on the tips results in tip end-face diameters of approximately $50-250 \mathrm{~nm}$. The consistently high resolution obtained with metal-coated tips is due to small aspherities on the tip apex resulting from the coating process.

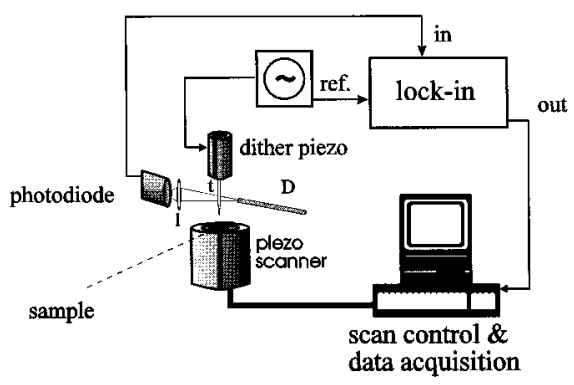

FIG. 1. Schematic of shear-force microscope. $D$ : detection fiber; $l$ : lens; $t$ : SNOM fiber tip. 


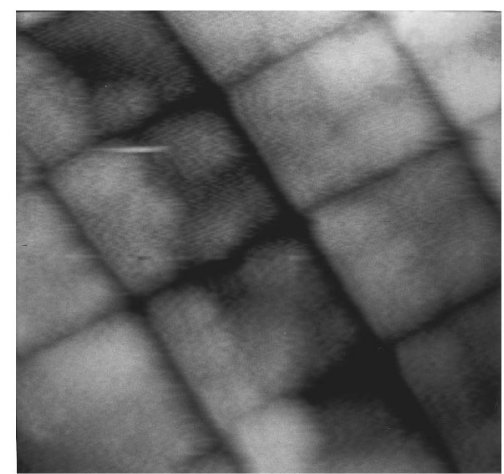

FIG. 2. Shear-force image of carbon grating test structure, taken with coated fiber tip. Scan size $=1.2 \mu \mathrm{m} \times 1.2 \mu \mathrm{m}$.

Scanning electron microscope (SEM) analysis of tips revealed that these aspherities can have radii of curvature lower than $20 \mathrm{~nm}$.

Figure 3 shows a shear-force image of a glass cover-slip surface. This image was taken using an uncoated tip, and the beam deflection method. This image demonstrates the resolution capability of such a setup. The protrusions on the surface have mean heights of 8-9 $\mathrm{nm}$, with lateral dimensions as small as $25 \mathrm{~nm}$. The resolution is limited by the tip shape rather than the shear-force dither amplitude.

\section{SHEAR-FORCE APPROACH CHARACTERISTICS}

In this section, the dependence of the amplitude of the SNOM fiber oscillations damped by the shear-force upon the distance between the fiber and the sample is analyzed. These dependences are called shear-force approach curves.

The vertical distance at which the fiber oscillations start to become damped out increases with increasing initial oscillation amplitude. Here, the damping distance is defined as the difference in tip-sample separation between which the fiber oscillation amplitude varies from $90 \%$ to $10 \%$ of its original, undamped value as the fiber approached the sample. The relationship between the damping distance and the initial oscillation amplitude is approximately linear, as illustrated in Fig. 4, which shows results measured for coated tips on a carbon surface.

The purpose of using shear-force distance regulation is to be able to maintain the distance between the probe and the sample at a constant value. To verify whether this was the case, approach curves were measured on three more types of

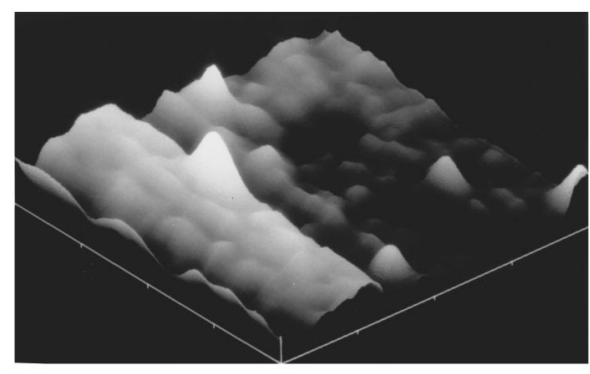

FIG. 3. Shear-force image of glass cover-slip surface, taken with uncoated tip. Scan size $=310 \mathrm{~nm} \times 310 \mathrm{~nm}$.

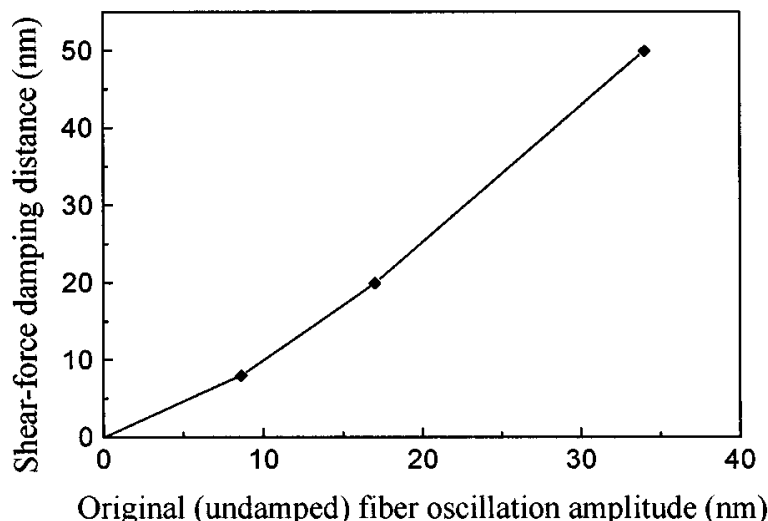

FIG. 4. Dependence of shear-force damping distance on original fiber oscillation amplitude, for a coated tip and a carbon sample.

flat surface: glass, aluminum, and chromium. These approach curves were measured for all three materials using both coated and uncoated tips. The results are summarized in Table I.

These distances were measured by taking five tips of both kinds, and by measuring approach curves for each one in at least six different places on each material type. The numbers above represent the average values measured. The standard deviation is $\sim 1 \mathrm{~nm}$. In some cases, the approach curves showed double steps, as shown in Fig. 5. The occurrence of such type of approach curve is less than $10 \%$. The curve may be explained by assuming a double tip, where both tips are vertically displaced. During approach, the tip nearest the sample experiences a shear-force damping first, followed by the second tip when it approaches close enough to the sample. The step in the approach curve is the point at which the second tip contributes to the damping. Due to the low observed occurrence of such a double step, penetration of a contamination layer on the surface can be ruled out here as being a cause.

A recent article $^{7}$ considers the shear-force interaction as basically being a short-range knocking between tip and sample. According to this work, the damping distance should depend on the initial oscillation amplitude of the fiber tip in an approximately linear fashion. This is consistent with the results shown in Fig. 4. Other major factors determining the damping distance are the angle between the tip axis and the normal to the surface, and the tip geometry. The larger the angle, the larger the corresponding damping distance. This may explain why the damping distance is different for different tips on similar samples. SEM studies of tips revealed that often the axis of the very end taper of an SNOM tip is not colinear with the rest of the tip. The extent is different for

TABLE I. Shear-force damping distances for different materials for coated and uncoated tips.

\begin{tabular}{cccc}
\hline \hline & Glass & Aluminum & Chromium \\
\hline Uncoated tips & $2.5 \mathrm{~nm}$ & $5 \mathrm{~nm}$ & \\
Coated tips & $4.5 \mathrm{~nm}$ & $10 \mathrm{~nm}$ & $3 \mathrm{~nm}$ \\
\hline \hline
\end{tabular}




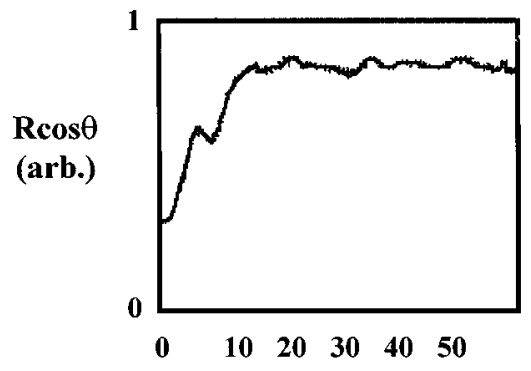

Tip-sample separation (nm)

FIG. 5. Shear-force approach curve showing double step.

different tips; in some cases the end taper is at $30^{\circ}-50^{\circ}$ to the rest of the tip. Such tips are not actually used for measurements, but there could easily be up to $10^{\circ}-15^{\circ}$ variation between the tips used. For imaging, it is the main axis of the fiber that is aligned to the sample surface normal. However, this work offers no insight into the fact that different areas of a sample exhibit different damping distances when the only difference is the material type. We shall return to this point later. If image charge current dissipation were the sole mechanism, then the effect should depend only on the conductivity of the sample. This is clearly not so. A recent work suggested a link between the damping distance and the hydrophobicity/hydrophilicity of the various constituents of the sample. ${ }^{11}$ Either way, there are likely to be at least two mechanisms involved.

\section{SHEAR-FORCE IMAGING OF SAMPLES CONTAINING TWO MATERIALS}

The fact that different materials have different damping distances means that, while imaging a sample containing different materials, one is not imaging at constant height. This is graphically illustrated in Fig. 6. This figure shows typical approach curves for both glass and aluminum. The values along the $x$-axis cannot be guaranteed to be the actual values of the separation between the end of the tip and the surface. It is of course possible that the oscillations are damped completely when a finite separation, say $1 \mathrm{~nm}$, is left. However, this does not change anything in the above definition of damping distance, or in the analysis which follows.

For shear-force imaging, the working distance is usually chosen to be that distance at which $R \cos \theta$ reaches half or so of its initial value. From Fig. 6, this corresponds to working distances of approximately 2.3 and $5 \mathrm{~nm}$ for glass and aluminum, respectively, if $R \cos \theta$ is maintained at $50 \%$ of the initial undamped value. To demonstrate the consequences of this common approach, a 4-nm film of aluminum was evaporated onto half of one side of a glass cover slip. The other half was left uncoated. A series of images was taken of a particular area of this sample, at different working distances. These were set by changing the set value of $R \cos \theta$. Figure 7 shows three images taken for this set value to be (a) $85 \%$, (b) $70 \%$, and (c) $50 \%$ of the initial value. The corresponding line profiles show that the apparent thickness of the aluminum island varies from 8 to 7.4 to $6.3 \mathrm{~nm}$, approaching the

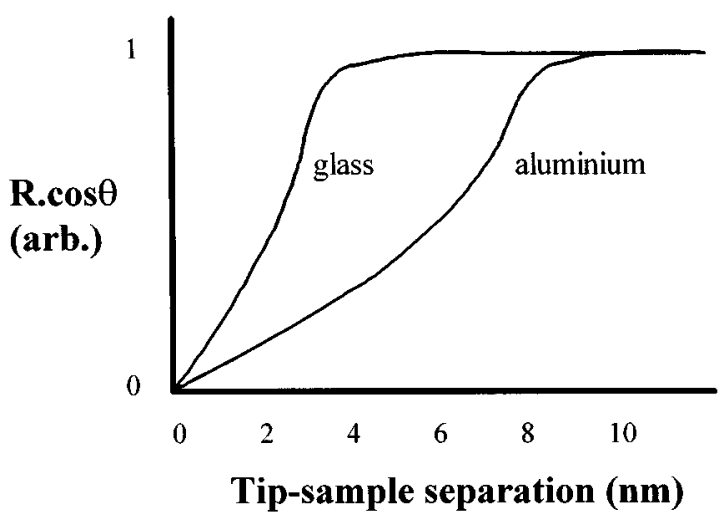

FIG. 6. Illustration of shear-force approach curves for a coated tip on glass and aluminium samples.

actual value as the working distance approaches zero. This is fully consistent with Fig. 6 . Thus, if one wants to work at truly constant height, they must work at or just before contact. However, this would subject the tip to large shearing forces, causing the coating to flake off and the tip to degrade, shortening the lifetime. Also, the close proximity to the sample would greatly increase the chances of tip crashes, which will also degrade the tip. One possible solution would be to have a setup whereby the tip oscillation amplitude is only a few $\AA$, giving also a working distance of a few $\AA$. In this case, the forces exerted on the tip could be reduced. The problem of tip crashes still remains, however. In order to be able to scan reasonably large areas with such small working distances, a fast feedback loop is required, as are high tip resonance frequencies. Unfortunately, the higher the resonance frequency, the higher the force required to damp the oscillations. Hence, there is a compromise between scan speed, tip durability, and accuracy of topographic measurements.

The following experimental results prove that shearforce is not caused by a sole physical mechanism, but it is rather a combination of at least two mechanisms. It is shown here that the value of the shear-force damping depends (1) upon the lateral size of electrically conducting features on a dielectric surface, and (2) upon the nature of the tip. However, this dependence is shown to be much more drastic for the second of the two mechanisms. We therefore believe that one of the mechanisms is likely to be image current damping (or at least electrical in nature), which strongly depends on the local resistivity of the surface features. Image current damping could be crudely thought of in the following manner: the tip, which is several mm long and sharp, could be considered a lightning rod. It will accumulate charge at its apex. The tip, when placed in close proximity to a surface, will induce charge on the surface. There will result a small attractive force between tip and sample. Due to the extremely large normal spring constant of the tip, this force will produce negligible effect apart maybe from slight deformation of tip and sample. The dithering of the tip, however, can give rise to currents in tip and sample which effectively damp the tip's motion. A simple estimate gives that a charge of $\sim 10^{-17} \mathrm{C}$ produces a tip-sample force of $\sim 1 \mathrm{nN}$ at a tip- 

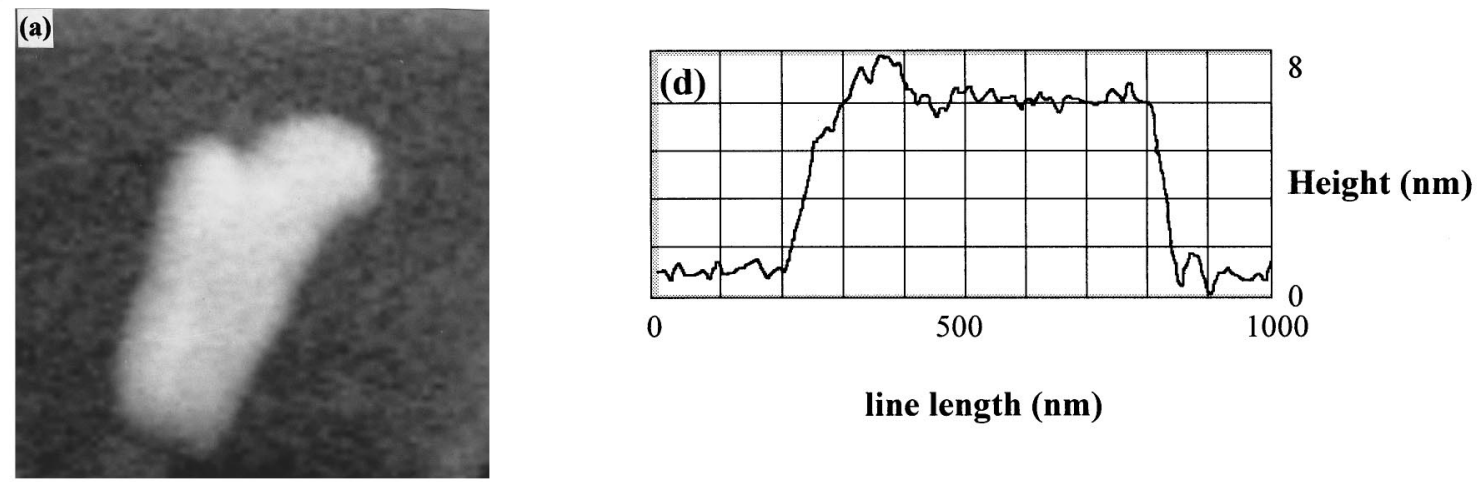

line length (nm)
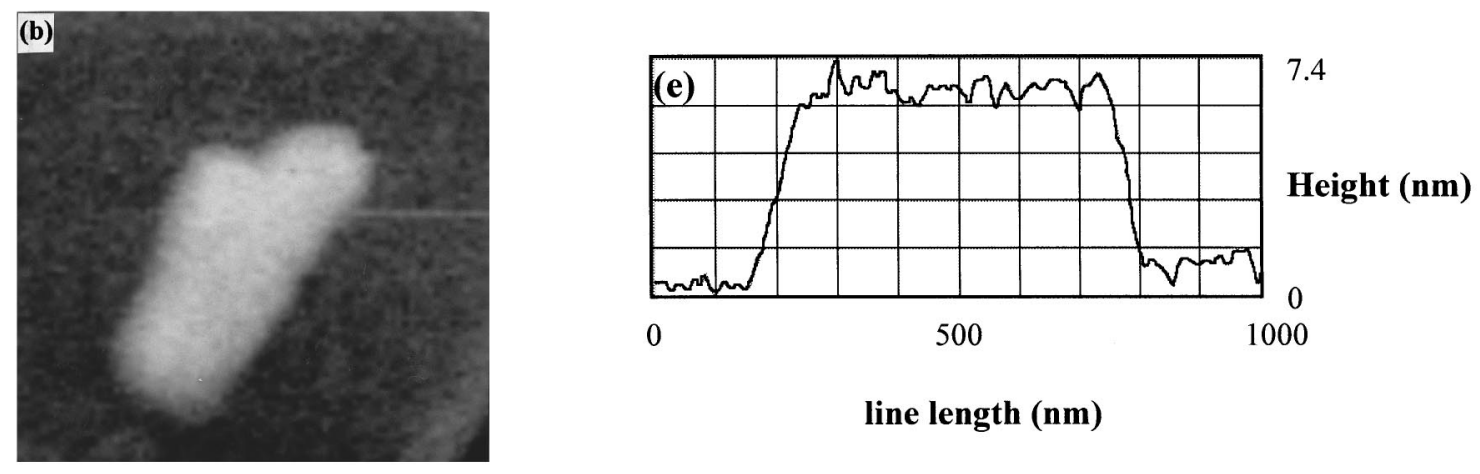

line length (nm)
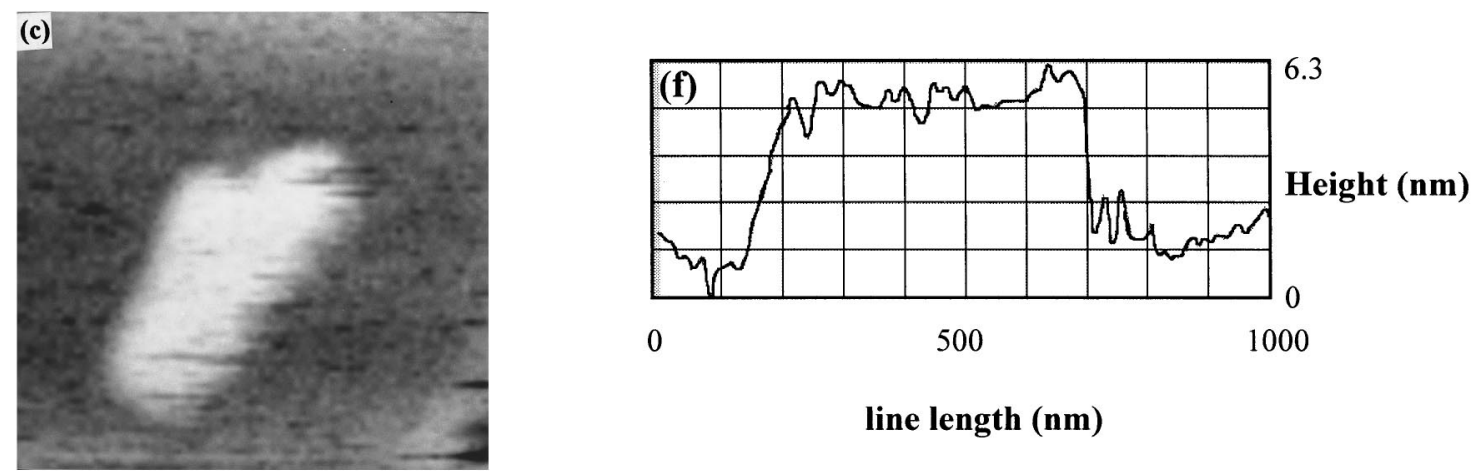

line length (nm)

FIG. 7. Images of aluminium grain on glass for three different shear-force damping set-points; (a) $85 \%$; (b) $70 \%$; (c) $50 \%$ of original fiber oscillation amplitude. Scan sizes $=700 \mathrm{~nm} \times 700 \mathrm{~nm}$.

sample separation of $\sim 1 \mathrm{~nm}$. Alternatively, the charging could be a result of the dithering, due to friction between the tip and sample.

The sample studied in these experiments was an aluminum pattern on glass grown by evaporation on the glass partly shadowed by latex spheres. The thickness of the film is $30 \mathrm{~nm}$. The sample consists of islands of aluminum film with the lateral size of the islands ranging mainly from 45 to over $200 \mathrm{~nm}$. The thickness of the film in the center of all the islands is of course the same. Once the lateral extent of the islands decreases below approximately $25-30 \mathrm{~nm}$, the thickness in the center should decrease below $30 \mathrm{~nm}$ due to shadowing by the latex spheres and the finite size of the evaporation source. Thus, all the islands studied here should have the same central height. For the shear-force image of this sample, shown in Fig. 8, the free oscillation amplitude of the fiber was set at approximately $30 \mathrm{~nm}$, and the set point, or reference amplitude, was approximately $15 \mathrm{~nm}$. The working distances for a coated tip under these conditions are $18 \mathrm{~nm}$ for aluminum and $10 \mathrm{~nm}$ for glass. There is clearly a shadow or double image of the main area of the film on its right hand edge. This indicates the presence of a double tip. Images obtained with double tips have been observed before, in scanning tunneling microscopy (STM) and atomic force microscopy (AFM) ${ }^{12}$ However, none of the small islands is imaged with a double edge. Compare, for example, the place A on the edge of the main area with the island B. The apparent height of both of these areas is the same, as measured from height profiles. However, the island B clearly shows no double edge. This is further illustrated in Fig. 9, which is a zoom-in of part of Fig. 8. We can therefore exclude any tip change between the two points of the imaged area A and B, since the double tip remains in place for the whole duration of the scan on this image. The scan direction is along the $x$-axis, and the double tip clearly remains active until the last line of the scan. Therefore, we must conclude that there are 


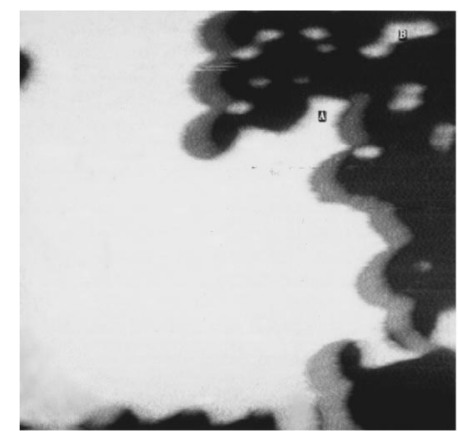

FIG. 8. Shear-force image of aluminium pattern on glass showing double edge. Scan size $=2.4 \mu \mathrm{m} \times 2.4 \mu \mathrm{m}$.

two different shear-force mechanisms sensed by the two tips or, rather, at least one mechanism experienced differently by both tips: one is effective on the large and also on the small aluminum islands (tip 1). The other (tip 2) is only effective on the large areas but not on the small islands. We could therefore suggest that the mechanism(s) are to some extent dependent on the effective local conductivity of the sample, and could result from image-current damping. The two tips are sensitive to the two different mechanisms due to either (i) having different shapes; or more likely (ii) being made of different materials, e.g., glass and aluminum. Figure 10 is a height profile through the line illustrated in Fig. 9. The height profile of the edge of the main area of the film indicates that the height difference between both tips is 22.5 $\mathrm{nm} \pm$ the working height difference between the two tips on the main aluminum film.

In order to analyze this situation, the various damping distances are denoted by the form $d_{x y}$, where $x=1,2$, for tip 1 and tip 2; and $y=g$ for glass, $\mathrm{Al}$ for aluminum, and $i$ for aluminum island. The actual height of the aluminum film is called $h$. An analysis of apparent height $h_{2}$ is carried out with relation to the medium-sized aluminum islands.

By reference to Figs. 10 and 11, the following relations apply:

$$
\begin{aligned}
h_{3}= & h+d_{1 \mathrm{al}}-d_{1 g}, \\
& \text { i.e., } 30 \mathrm{~nm}+18 \mathrm{~nm}-10 \mathrm{~nm}=38 \mathrm{~nm}, \\
h_{2}= & h+d_{1 \mathrm{i}}-d_{1 \mathrm{~g}}, \quad \text { i.e., } d_{1 \mathrm{i}}=7 \mathrm{~nm} .
\end{aligned}
$$

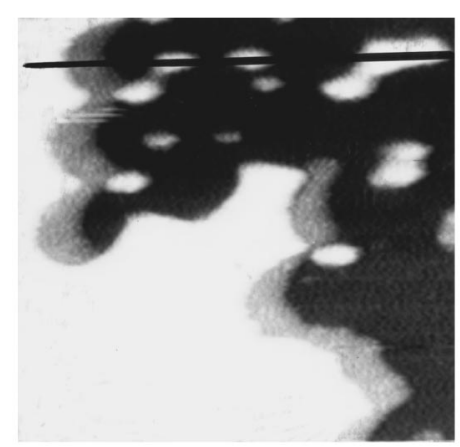

FIG. 9. Zoom-in of part of Fig. 8. Scan size $=1.2 \mu \mathrm{m} \times 1.2 \mu \mathrm{m}$.

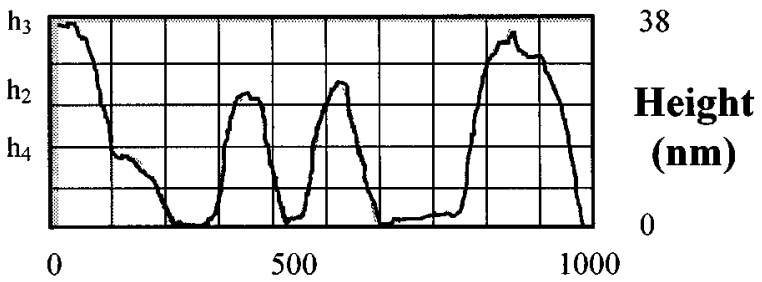

Tip position along line (nm)

Consequently, the working distance of tip 1 above the aluminum islands is $8 \mathrm{~nm}, 10 \mathrm{~nm}$ for glass, and $18 \mathrm{~nm}$ for the main aluminum film. For this reason, the measured height of the islands is $2 \mathrm{~nm}$ less than the actual value, and that of the main $\mathrm{Al}$ film is $8 \mathrm{~nm}$ greater than the actual value.

The next relations are with regard to tip 2, i.e.,

$$
h_{3}-h_{4}=d+d_{1 \mathrm{al}}-d_{2 \mathrm{al}} \text {, i.e., } d=d_{2 \mathrm{al}}+2.5 \mathrm{~nm} \text {. }
$$

The fact that there are no double images for the aluminum islands means that tip 2 does not approach close enough to the islands to be within its damping distance. Thus,

$$
d+d_{1 \mathrm{~g}}>h+d_{2 \mathrm{i}} \text {, i.e., } d-d_{2 \mathrm{i}}>20 \mathrm{~nm} \text {. }
$$

In combination with Eq. (3), this means that

$$
d_{2 \mathrm{al}}-d_{2 \mathrm{i}}>17.5 \mathrm{~nm} \text {. }
$$

While there is not enough information regarding the values of the working distances for tip 2, from the example of tip 1 Eq. (5) is not unreasonable. The above values for damping distances will vary for both tips for different islands. Figure 12 shows a plot of the measured apparent heights of the aluminum islands as a function of the islands' area. The fact that there is no double image for the large aluminum island while its measured height is the same as that of the main area of the film indicates that the two tips must be made of different materials.

As to why there are any variations between damping distances for a given tip and different samples, the knocking mechanism $^{7}$ does not explain this. On the basis of this model, one could possibility be related to the roughness of different surfaces; i.e., an $\mathrm{Al}$ film on glass may be rougher than the glass substrate. Consequently, the tip should see a larger variation in contact angle when scanning above $\mathrm{Al}$ than above glass. This may explain the different average damping distances. However, on the basis of a large number

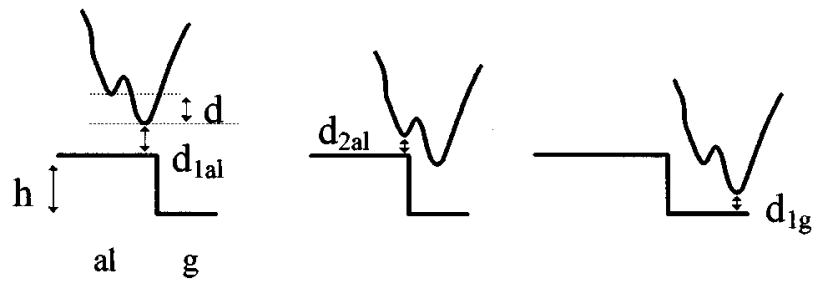

FIG. 11. Illustration of double-tip scheme. 


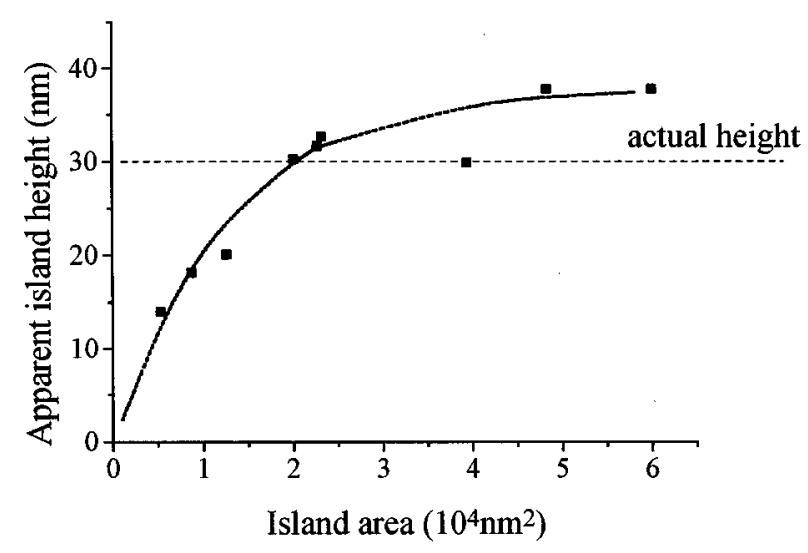

FIG. 12. Plot showing dependence of apparent height of aluminium islands on the islands' area.

of images obtained during the course of this work, there is no real evidence to support this. ${ }^{13}$ The images shown in Figs. 8 and 9 demonstrate that, for these samples, the surface roughness for $\mathrm{Al}$ and glass is comparable to better than $1 \mathrm{~nm}$. Therefore, there must be an extra mechanism, related to the nature of the contact between tip and sample. The results shown here indicate that the damping distance may partly depend on local conductivity. However, this still does not explain the fact that the damping distance for $\mathrm{Cr}$ is less than that for glass. Clearly the shear-force mechanism is more complex, and, while the idea of image-current damping partly ties in with the above results, it is likely that the chemical properties of the sample — such as hydrophilicityalso play a role here.

The fact that shear-force characteristics are essentially unchanged in vacuum and in liquid He tends to reduce the possibility of contamination layers being a primary mechanism. Nonetheless, as these experiments were conducted in air, there is bound to be a thin water/contaminant film covering the entire sample. This would be somewhat of a benefit, as tip-sample adhesion forces are reduced by the presence of the film. ${ }^{12}$ Of course, it is possible that the nature of the film is determined by the sample type, i.e., the film may be structurally different on $\mathrm{Cr}$ than on $\mathrm{Al}$ or glass due to the chemical nature (hydrophobicity, crystal structure) of these materials. This possibility should be considered in the future: it is possible that in shear-force imaging, the damping is partly due to viscous damping in a contaminant layer. If one images at the onset of damping, they will essentially image the shape of this layer. If one then images closer to the sample, almost at contact, they will image the shape of the sample. If the above idea regarding the form of this layer is correct, both images will be different. This is consistent with the results shown in Fig. 7, but cannot explain the results of Figs. 8-12. The question that needs more attention here is: If the damping is due to a contaminant layer, why are double steps not observed more often in shear-force approach curves-one from penetration of this layer and one from contact with the sample? In order to resolve these questions, shear-force characteristics should be measured for different materials in vacuum.

\section{CONCLUSIONS}

There are many possible artifacts of a fundamental nature involved in shear-force imaging. Therefore, it is not a reliable technique for accurate topographic measurements. It was shown that different materials have different shear-force damping characteristics and islands of conducting materials have different damping characteristics determined by the size of the islands. Shear-force damping was shown to be a result of at least two different mechanisms of interaction between the tip and the sample, one of which may be image current damping, and another which may be a knocking mechanism.

${ }^{1}$ E. Betzig, J. K. Trautman, T. D. Harris, J. S. Weiner, and R. L. Kostelak, Science 251, 1468 (1991).

${ }^{2}$ D. W. Pohl, W. Denk, and M. Lanz, Appl. Phys. Lett. 44, 651 (1984).

${ }^{3}$ D. Courjon and C. Bainier, Rep. Prog. Phys. 57, 989 (1994).

${ }^{4}$ C. Durkan and I. V. Shvets, Ultramicroscopy 61, 227 (1995).

${ }^{5}$ G. A. Massey, Appl. Opt. 658 (1984).

${ }^{6}$ R. Toledo-Crow, P. C. Yang, Y. Chen, and M. Vaez-Iravani, Appl. Phys. Lett. 60, 2957 (1992).

${ }^{7}$ M. K. Gregor, P. G. Blome, J. Schöfer, and R. G. Ulbrich, Appl. Phys. Lett. 68, 307 (1996).

${ }^{8}$ C. Durkan and I. V. Shvets, J. Appl. Phys. 79, 1219 (1996).

${ }^{9}$ F. F. Froehlich and T. D. Milster, Appl. Opt. 34, 7273 (1995).

${ }^{10}$ TEM test sample code S-104, Agar Scientific Ltd., U.K.

${ }^{11}$ Results presented by N. van Hulst at NFO-3 Conference on Near-Field Optics, Brno, Czech Republic, May 9-11, 1995.

${ }^{12}$ D. A. Walters, D. Hampton, B. Drake, H. G. Hansma, and P. K. Hansma, Appl. Phys. Lett. 65, 787 (1994).

${ }^{13}$ C. Durkan, Ph.D. thesis, Trinity College, Dublin, 1996. 